\title{
Antimicrobial resistance, multilocus sequence types and virulence profiles of ESBL producing and non-ESBL producing uropathogenic Escherichia coli isolated from cats and dogs in Switzerland
}

\author{
Zogg, Anna Lena ; Zurfluh, Katrin ; Schmitt, S ; Nüesch-Inderbinen, Magdalena ; Stephan, Roger
}

\begin{abstract}
Among 64 uropathogenic Escherichia coli (UPEC) isolated from 13 cats and 51 dogs, 35 were extended-spectrum beta-lactamase (ESBL) producers, and 29 were non-ESBL producers. Forty-six $(71.9 \%)$ of the isolates were multidrug resistant (MDR). Among the ESBL producers, blaCTX-M-15 ( $\mathrm{n}=$ $17 / 48.6 \%$ of the blaESBLs), blaCTX-M-1 ( $\mathrm{n}=10 / 28.6 \%)$, blaCTX-M-55 ( $\mathrm{n}=4 / 11.4 \%)$, blaCTX-M-14 $(\mathrm{n}=3 / 8.6 \%)$, and blaCTX-M-27 $(\mathrm{n}=1 / 2.9 \%)$ were identified. The plasmid-mediated fluoroquinolone resistance genes aac $\left(6^{\prime}\right)-\mathrm{Ib}-\mathrm{cr}$, qnrB and the azithromycin resistance gene $\mathrm{mph}(\mathrm{A})$ were detected in 17 (26.6\% of all isolates), one $(1.6 \%)$ and in $13(20.3 \%)$ respectively. The most frequent phylogenetic groups were $\mathrm{C}(\mathrm{n}=19)$ and B2 $(\mathrm{n}=15)$. Twenty-six different sequence types (STs) were identified, with two being novel. The most frequent STs were ST410 $(\mathrm{n}=16 / 25 \%)$, ST131, and ST73 (both $\mathrm{n}=5 / 7.8 \%$ ), and ST361 ( $\mathrm{n}=4 / 6.3 \%)$. Ten $(15.6 \%)$ of the STs have been associated with urinary tract infection (UTI) in humans, suggesting zoonotic potential. Among seven virulence-associated genes, fyuA was the most prevalent. The overall aggregate virulence factor (VF) score was highest for isolates belonging to phylogenetic group B2 (median aggregate VF score 6, mean score 5,5, range 3-7), and lowest for isolates belonging to phylogenetic group C (0/ 0.5/0-3). The most frequent ST in this study, ST410, harboured the lowest number of VF (0/0,3/0-2). VF scores were higher in NDR (4/3.8/3-4) than in MDR $(1 / 1,9 / 0$ $7)$, and higher in non-ESBL producing isolates $(3 / 3 / 0-7)$ than in ESBL producers $(1 / 1,7 / 0-7)$. Our data advance our knowledge of the phenotypic and genotypic characteristics of UPEC in companion animals and their potential for infection, zoonotic transmission and dissemination of antimicrobial resistance determinants.
\end{abstract}

DOI: https://doi.org/10.1016/j.vetmic.2018.02.011

Posted at the Zurich Open Repository and Archive, University of Zurich ZORA URL: https://doi.org/10.5167/uzh-167650

Journal Article

Accepted Version

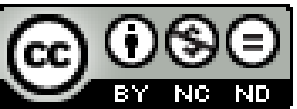

The following work is licensed under a Creative Commons: Attribution-NonCommercial-NoDerivatives 4.0 International (CC BY-NC-ND 4.0) License.

Originally published at:

Zogg, Anna Lena; Zurfluh, Katrin; Schmitt, S; Nüesch-Inderbinen, Magdalena; Stephan, Roger (2018). Antimicrobial resistance, multilocus sequence types and virulence profiles of ESBL producing and non- 
ESBL producing uropathogenic Escherichia coli isolated from cats and dogs in Switzerland. Veterinary Microbiology, 216:79-84.

DOI: https://doi.org/10.1016/j.vetmic.2018.02.011 
1 Antimicrobial Resistance, Multilocus Sequence Types and Virulence Profiles of ESBL

2 producing and non-ESBL producing uropathogenic Escherichia coli isolated from Cats

3 and Dogs in Switzerland

4

5

Anna Lena Zogg a, Katrin Zurfluh ${ }^{\text {a }}$, Sarah Schmitt ${ }^{\mathrm{b}}$, Magdalena Nüesch-Inderbinen ${ }^{\text {a }}$, Roger

$6 \quad$ Stephan $^{\text {a* }}$

7

8

9

${ }^{a}$ National Centre for Enteropathogenic Bacteria and Listeria, Institute for Food Safety and

Hygiene, Vetsuisse Faculty University of Zürich, Zürich, Switzerland

11

12

${ }^{\mathrm{b}}$ Institute of Veterinary Bacteriology, Vetsuisse Faculty, University of Zürich, Switzerland

*Corresponding author:

Roger Stephan, Institute for Food Safety and Hygiene, Vetsuisse Faculty, University of

Zurich, Winterthurerstrasse 272, CH-8057 Zurich, Switzerland. 


\section{Abstract}

Among 64 uropathogenic Escherichia coli (UPEC) isolated from 13 cats and 51 dogs, 35 were extended-spectrum beta-lactamase (ESBL) producers, and 29 were non-ESBL producers. Forty-six (71.9\%) of the isolates were multidrug resistant (MDR). Among the ESBL producers, $b l a_{\mathrm{CTX}-\mathrm{M}-15}\left(\mathrm{n}=17 / 48.6 \%\right.$ of the bla $\left._{\mathrm{ESBLs}}\right)$, bla $_{\mathrm{CTX}-\mathrm{M}-1}(\mathrm{n}=10 / 28.6 \%)$, bla $_{\mathrm{CTX}-\mathrm{M}-55}$ $(\mathrm{n}=4 / 11.4 \%)$, bla $_{\mathrm{CTX}-\mathrm{M}-14}(\mathrm{n}=3 / 8.6 \%)$, and $\operatorname{bla}_{\mathrm{CTX}-\mathrm{M}-27}(\mathrm{n}=1 / 2.9 \%)$ were identified. The plasmid-mediated fluoroquinolone resistance genes $a a c\left(6^{\prime}\right)-I b-c r, q n r B$ and the azithromycin resistance gene $m p h(A)$ were detected in $17(26.6 \%$ of all isolates), one (1.6\%) and in 13 (20.3\%) respectively. The most frequent phylogenetic groups were $C(n=19)$ and $B 2(n=15)$. Twenty-six different sequence types (STs) were identified, with two being novel. The most frequent STs were ST410 (n=16/25\%), ST131, and ST73 (both n=5/7.8\%), and ST361 $(n=4 / 6.3 \%)$. Ten $(15.6 \%)$ of the STs have been associated with urinary tract infection (UTI) in humans, suggesting zoonotic potential. Among seven virulence-associated genes, $f y u A$ was the most prevalent. The overall aggregate virulence factor (VF) score was highest for isolates belonging to phylogenetic group B2 (median aggregate VF score 6, mean score 5,5, range 37), and lowest for isolates belonging to phylogenetic group $\mathrm{C}(0 / 0.5 / 0-3)$. The most frequent ST in this study, ST410, harboured the lowest number of VF $(0 / 0,3 / 0-2)$. VF scores were higher in NDR (4/3.8/3-4) than in MDR (1/1,9/0-7), and higher in non-ESBL producing isolates $(3 / 3 / 0-7)$ than in ESBL producers $(1 / 1,7 / 0-7)$. Our data advance our knowledge of the phenotypic and genotypic characteristics of UPEC in companion animals and their potential for infection, zoonotic transmission and dissemination of antimicrobial resistance determinants.

1 Key words: uropathogenic, Escherichia coli, cats, dogs, MLST, virulence 


\section{Introduction}

Escherichia coli occurs naturally in the digestive tract of humans and animals as a commensal, however, some strains of $E$. coli can cause extraintestinal diseases, such as pneumonia, sepsis, meningitis and urinary tract infections (UTI) (Kaper et al., 2004).

Commensal strains have been shown by phylogenetic analyses to be associated with phylogenetic group A, B1 or C, while virulent extraintestinal E. coli strains belong mainly to group B2 and, to a lesser extent, to group D, E, or F (Clermont et al., 2013).

Uropathogenic E. coli (UPEC) is the most common etiologic agent of UTI in humans and animals (Foxman, 2010; Olin and Bartges, 2015). The ability of UPEC to cause disease is associated with the expression of specific virulence factors (VF) encoding adhesins, invasins, toxins and other proteins (Kaper et al., 2004).

Among companion animals, E. coli clones that are found in the intestine may cause urinary tract infection (UTI) (Johnson et al., 2008b). Furthermore, UPEC may be acquired via external reservoirs: previous studies have demonstrated that humans and their companion animals can share pathogenic types of E. coli, including UPEC, indicating zoonotic as well as anthroponotic transmission (Johnson et al., 2008b; Johnson et al., 2008c; Johnson et al., 2009). Other significant sources of pathogenic E. coli include raw meat canine diets and raw or inadequately cooked chicken meat (Glaser 2012; Schmidt et al., 2015).

For uncomplicated UTI in cats and dog, the first-line therapeutic option is amoxicillin, with sulfamethoxazole/trimethoprim and fluoroquinolones remaining appropriate for complicated infections and pyelonephritis, respectively (Barsanti, 2012). The emergence of antimicrobial resistance among UPEC is of great concern and increases the risk of antimicrobial treatment failure in companion animals, as in humans.

Understanding the prevalence of antimicrobial resistance and pathogenicity of UPEC isolated from cats and dogs is important both from veterinary medicine and public health perspectives, 
but information regarding the population structure and virulence association of UPEC from cats and dogs is still limited.

The aim of this study was therefore to (i) assess the antimicrobial susceptibility of uropathogenic E. coli isolates obtained throughout 2012-2016 from Swiss dogs and cats, (ii) to detect and characterize plasmid-mediated resistance to third-generation cephalosporins, fluoroquinolones and azithromycin, (iii) to characterize the strains by phylogenetic grouping, multilocus sequence typing (MLST) and virulence profiling.

\section{Materials and Methods}

\subsection{Bacterial isolates}

From a total of 205 Escherichia coli strains isolated between 2012 and 2016 from urine samples of companion animals admitted to the University of Zürich veterinary clinic, a total of 64 (35 ESBL-producing and 29 randomly selected non-ESBL producing) strains from 13 cats and 51 dogs were analysed. Strain identification and preliminary antimicrobial susceptibility profiling was performed using VITEK 2 Compact system with AST GN38 cards (Biomérieux, Marcy l'Etoile, France) according to the manufacturer's instructions.

\subsection{Phenotypic and genotypic antimicrobial resistance testing}

Antimicrobial susceptibility testing was performed using the disk-diffusion method and the antibiotics ampicillin (AM), cefazolin (CZ), cefotaxime (CTX), amoxicillin with clavulanic acid (AMC), cefepime (FEP), nalidixic acid (NA), ciprofloxacin (CIP), sulfamethoxazole/trimethoprim (SXT), fosfomycin (FOS), nitrofurantoin (F/M), streptomycin (S), kanamycin $(\mathrm{K})$, gentamicin $(\mathrm{G})$, chloramphenicol (C) and tetracycline (TE) (Becton 
105 Dickinson, Allschwil, Switzerland). Results were interpreted according to Clinical and

106 Laboratory Standards Institute (CLSI) performance standards (CLSI, 2016). Isolates

107 displaying resistance to three or more classes of antimicrobials (counting beta-lactams as one

108 class) were defined as multidrug-resistant (MDR). Synergistic effects between AMC and

109 CTX and FEP were regarded as an indication of the presence of an ESBL producer (Kaur et

110 al., 2013).

111 For genotypic analyses, bacterial DNA was extracted using a standard heat lysis protocol. The

112 presence of $b l a_{\mathrm{ESBL}}$ genes belonging to the $b l a_{\mathrm{TEM}}, b l a_{\mathrm{SHV}}$, and $b l a_{\mathrm{CTX}-\mathrm{M}}$ families was

113 confirmed by PCR and amplicons were sequenced as described previously (Woodford et al.,

114 2006; Zurfluh et al., 2015). Ciprofloxacin resistant strains were examined for mutations in

115 quinolone resistance-determining regions (QRDRs) of gyrA and $\operatorname{parC}$, using previously

116 described PCR and sequencing primers (Zurfluh et al., 2014). Screening all isolates for the

117 plasmid-mediated fluoroquinolone resistance genes $a a c\left(6^{\prime}\right)-I b-c r, q n r A, q n r B, q n r C, q n r D$,

$118 q n r S$, and qepA was carried out as described previously (Zurfluh et al., 2014). Further, all

119 strains were screened for the plasmid-mediated azithromycin resistance gene $m p h(A)$ using

120 previously described primers (Ojo et al., 2004). DNA samples from isolates described

121 previously were used as positive controls (Nüesch-Inderbinen et al., 2017; Zurfluh et al.,

122 2014).

123 Synthesis of primers and DNA custom sequencing was carried out by Microsynth (Balgach,

124 Switzerland) and nucleotide sequences were analysed with CLC Main Workbench 6.6.1. For

125 database searches the BLASTN program of NCBI (http://www.ncbi.nlm.nih.gov/blast/) was

126 used.

\subsection{Phylogenetic characterization and multilocus sequence typing}

130 The distribution of phylogenetic groups amongst the isolates was determined by the 
131 improved phylotyping PCR approach described by Clermont et al., targeting the genes chuA,

$132 y j a A, \operatorname{arp} A$ and TspE4.C2 (Clermont et al., 2013). Isolates were thereby classified as

133 belonging to one of the eight phylogenetic groups A, B1, B2, C, D, E, F, (E. coli sensu

134 stricto), or Escherichia clade I.

135 For multilocus sequence typing of E. coli isolates, internal fragments of the seven

136 housekeeping genes ( $a d k$, fum $C, \operatorname{gyr} B, i c d, m d h$, purA, and $\operatorname{rec} A)$ were amplified by PCR

137 from DNA, as described by Wirth et al. (Wirth et al., 2006). Sequencing of the amplification

138 products was performed by Microsynth (Balgach, Switzerland). Sequences were imported

139 into the E. coli MLST database website (http://mlst.ucc.ie/mlst/dbs/Ecoli) to determine

140 sequence types (STs). Alleles and STs that had not been previously described were designated

141 new ST, but not assigned numerical designations, since whole-genome sequencing was not

142 performed.

143

\section{2.4. Virulence factor (VF) determination}

146 All 64 strains were tested by conventional PCR for the presence of genes of VF that mediate

147 adhesion (p-fimbrial adhesion genes papAH and $p a p E F$, and the chaperone-usher fimbria

$148 y f c v)$, toxins $(\alpha$-haemolysin $h l y A)$, siderophores (the ferric yersiniabactin uptake protein $f y u A$ ),

149 serum resistance (traT), and the right-hand terminus of pathogenicity island (PAI) from $E$.

150 coli strain CFT073, using primers and conditions described previously (Johnson and Stell,

151 2000; Spurbeck et al., 2012). The aggregate VF score was defined as the number of unique

152 VF detected for each isolate, counting the PAI marker as one. 
157 The Based Upon Related Sequence Types (eBURST) clustering algorithm in phyloviz 158 (www.phyloviz.net) was used to visualise relationships between the isolates.

\section{Results}

161

\subsection{Antimicrobial resistance}

Between 2012-2016, 205 E. coli strains were collected (41 in 2012, 121 in 2013, 17 in 2014, 17 in 2015 and 9 in 2016). During these years, ESBL producers were detected in 10 (24.4\%), $12(9.9 \%), 4(23.5 \%), 6(35.3 \%)$, and $3(33.3 \%)$ of the isolates, respectively. Resistance profiles of the 35 ESBL producing and 29 non-ESBL producing isolates to all 15 antimicrobials tested are shown in detail in supplementary Table S1. Resistance rates to classes of antimicrobials commonly used for treating feline and canine E. coli UTIs are summarized in Table 1.

Overall, 46 (71.9\%) of the isolates were MDR (88.6\% of the ESBL-producers, and $51.7 \%$ of the non-ESBL producers, respectively). The presence of $b l a_{\mathrm{ESBL}}$ genes was confirmed and the genes were identified in 35 phenotypic ESBL producers as bla $a_{\text {СТХ-М-15 }}(\mathrm{n}=17 / 48.6 \%$ of the bla $\left._{\mathrm{ESBLS}}\right), \operatorname{bla}_{\mathrm{CTX}-\mathrm{M}-1}(\mathrm{n}=10 / 28.6 \%)$, bla $_{\mathrm{CTX}-\mathrm{M}-55}(\mathrm{n}=4 / 11.4 \%), \operatorname{bla}_{\mathrm{CTX}-\mathrm{M}-14}(\mathrm{n}=3 / 8.6 \%)$, and

$175 \operatorname{bla}_{\mathrm{CTX}-\mathrm{M}-27}(\mathrm{n}=1 / 2.9 \%)$. Except for one bla $a_{\mathrm{CTX}-\mathrm{M}-1}$, all bla $a_{\mathrm{ESBLs}}$ were detected in canine isolates.

176 Overall, $51(79.7 \%)$ of the isolates were resistant to nalidixic acid, and 47 thereof were

177 ciprofloxacin resistant (Table 1 and supplementary Table S1). Among the isolates displaying

178 ciprofloxacin resistance, all revealed chromosomal mutations that result in amino acid

179 substitutions in GyrA and ParC (Table 2 and supplementary Table S1). The plasmid-mediated

180 fluoroquinolone resistance genes $a a c\left(6^{\prime}\right)-I b-c r$ and $q n r B$ were detected in $17(26.6 \%$ of all 
181 isolates), and one (1.6\%) isolate, respectively, and were restricted to canine isolates (Table 3

182 and supplementary Table S1). The plasmid-mediated azithromycin resistance gene $m p h(A)$

183 was found in $13(20.3 \%)$ of the isolates. The majority $(n=15 / 88.2 \%)$ of the 17 isolates with

184 plasmid-mediated fluoroquinolone resistance and the majority $(n=34 / 69.4 \%)$ of the 49

185 isolates with chromosomally mediated quinolone resistance were ESBL producers (Table 3).

186 Only 4 (6.3\%) isolates were non-drug resistant (NDR) (supplementary Table S1).

187

\subsection{Phylogenetic groups and ST}

189

190 As shown in Table 3, the most prevalent phylogenetic group was $\mathrm{C}(\mathrm{n}=19 / 29.7 \%$ of all

191 isolates), followed by group B2 ( $n=15 / 23.4 \%), B 1(n=10 / 15.6 \%)$, group $A(n=9 / 14 \%)$, group

$192 \mathrm{~F}(\mathrm{n}=8 / 12.5 \%)$, and group $\mathrm{D}(\mathrm{n}=3 / 4.7 \%)$. None of the isolates belonged to phylogenetic group

193 E or clade I. Among feline isolates, phylogenetic group B2 predominated $(\mathrm{n}=7 / 53.8 \%$ of all

194 feline isolates), whilst among canine isolates, the majority ( $\mathrm{n}=18 / 35.3 \%$ ) belonged to group C.

195 MLST assigned the majority ( $\mathrm{n}=39 / 60.9 \%)$ of the isolates to 9 different clonal complexes

196 (CC): CC10 (n=3), CC12 (n=2), CC23 (n=17), CC73 (n=5) and CC131 (n=5), CC156 (n=1),

$197 \operatorname{CC} 354(\mathrm{n}=2), \operatorname{CC} 469(\mathrm{n}=1)$, and CC648 $(\mathrm{n}=3)$.

198 Twenty-six different STs were identified, the three most common represented by ST410

$199(\mathrm{n}=16 / 25 \%)$, ST131, and ST73 (both $n=5 / 7.8 \%)$. Less frequently occurring STs included 200 ST533 $(n=4 / 6.3 \%)$, and ST361, ST744 and ST648 (each n=3/4.7\%, respectively). All other

201 STs were represented by one or two isolates only (Table 3 and supplementary Table S1).

202 Two new STs were detected, which however were not assigned numerical designations by the 203 E. coli MLST database (http://mlst.warwick.ac.uk/mlst/dbs/Ecoli). Figure 1 illustrates

204 eBURST showing relationships between the isolates. The prevalence of the most common ST, 205 ST410, was variable, declining from $50 \%$ in 2012 to $1 \%$ in 2015 , with an increase to $33.3 \%$ 
observed for 2016. Of the 64 isolates, a total of 10 (15.6\%) belonged STs frequently associated with human UTI (ST73 and ST131).

The MDR, ESBL- and non-ESBL producers were distributed throughout phylogroups A, B1, $\mathrm{B} 2, \mathrm{C}, \mathrm{D}$ and $\mathrm{F}$.

\subsection{Distribution of VF genes}

Overall, 47 (73.4\%) of the isolates tested positive for one or more of the VF genes (Table 3). Among the 64 isolates, the prevalence of individual VF genes was $53.1 \%$ for $f y u A, 48.4 \%$ for traT, $34.4 \%$ for $y f c v$ and PAI, $23.4 \%$ for $h l y A, 20.3 \%$ for $p a p A H$, and $18.8 \%$ for $p a p E F$.

216 The overall median aggregate VF score was highest for isolates belonging to phylogenetic 217 group B2 (median aggregate VF score 6, mean score 5,5, range 3-7), followed by isolates 218 from phylogenetic group F (3/2,9/1-4). Isolates assigned to phylogenetic group D scored $2192 / 2 / 2-2$, and isolates of phylogenetic group A scored 1/1,8/ 0-6. The overall median aggregate

220 VF scores were lowest for isolates belonging to phylogenetic group $\mathrm{C}(0 / 0.5 / 0-3)$.

221 Among the most common STs in this study, ST131 harboured the highest number of VF 222 (7/6,4/4-7), followed by ST73 (4/4,6/4-7), and ST648 (4/3,7/3-4). Of the most prevalent ST in this study, ST410 harboured the lowest number of VF (0/0,3/0-2). VF scores were higher in NDR (4/3.8/3-4) than in MDR (1/1,9/0-7), higher in non-ESBL producing isolates (3/3/0-7) than in ESBL producers $(1 / 1,7 / 0-7)$, and higher in isolates that 226 did not harbour $m p h(A)(2 / 2,6 / 0-7)$, than those that harboured $m p h(A)(1 / 1,1 / 0-4)$.

\section{Discussion}

230 In this study, we assessed antimicrobial resistance, phylogenetic background, and virulence 231 profiles of ESBL producing, and non-ESBL producing UPEC isolated from cats and dogs 
admitted to the veterinary clinic of the University of Zürich, Switzerland. In veterinary as in

233 human medicine, antimicrobials are the cornerstone of treatment of bacterial UTI.

234 Sulfamethoxazole/trimethoprim, amoxicillin/clavulanate, quinolones and chloramphenicol are

235 antimicrobial compounds that are recommended for the treatment of feline and canine UTI

236 due to infection with E. coli (Barsanti, 2012). Our data show that infection management may

237 be compromised by the high rate of resistance to these drugs, similar to the situation /as

238 described for UPEC in humans (Gibreel et al., 2012; Nicolle 2013; Nolan et al., 2015;

239 Lavigne et al. 2016). Furthermore, of the 205 isolates from our strain collection, 35 (17\%)

240 were ESBL producers and the annual prevalence ranged from $9.9 \%$ to $35.3 \%$. This is a

241 remarkable increase compared to the prevalence of $7.5 \%$ ESBL producers among UPEC

242 isolated from companion animals at our institution between 2010-2011 (Huber et al., 2013),

243 and poses a threat to the efficacy of third-generation cephalosporins approved for use in

244 veterinary medicine, such as cefovecin (Stegemann et al., 2006). Further, the high prevalences

245 of $26.6 \% a a c\left(6^{\prime}\right)-I b-c r$ and $13.6 \% \mathrm{mph}(A)$ among the UPEC analysed in this study contrast

246 noticeably with the $2.3 \%$ and $13.6 \%$, respectively, observed for these genes in UPEC from

247 human primary care patients in Switzerland (Nüesch-Inderbinen et al., 2017), and indicate

248 that companion animals, particularly dogs, may serve as reservoirs for E. coli harbouring

249 these resistance genes.

250 In this study, we identified the phylogenetic groups based on the new Clermont method which

251 enabled, besides the phylogenetic groups A, B1, B2, and D, the identification of groups C and

252 F among the feline and canine UPEC. Our results show that in accordance to previous reports,

253 UPEC isolates from cats frequently belonged to group B2 (Wagner et al., 2014; Liu et al.,

254 2015). However, overall, phylogroup $\mathrm{C}$ had the highest prevalence, a finding which is in

255 contrast to previous studies of UPEC in animals as well as in humans that indicate the

256 predominance of extraintestinal, pathogenic group B2 (Barsanti, 2012; Gibreel et al., 2012).

257 Data on the prevalences of phylogroups C, E, F and clade I among UPEC are rare, but a 
recent study shows that among human isolates, approximately $25 \%$ belong to these groups

259 (Iranpour et al., 2015). Since $42.2 \%$ of the isolates belonged to the newly described

260 phylogroups $\mathrm{C}$ and $\mathrm{F}$, further studies are needed to provide a more refined understanding of

261 the prevalences of phylogenetic groups among E. coli isolates of animal and human origin.

262 Antimicrobial resistant as well as susceptible UPEC in humans are frequently associated with

263 specific clonal complexes such as CC69, CC73, CC95, ST127, and CC131 (Lau et al., 2008;

264 Gibreel et al., 2012). Some of these CCs or STs, such as CC73, ST127 and CC131 have also

265 been described as etiological agents of UTI in companion animals (Johnson et al., 2008a;

266 Johnson et al., 2009). Corresponding well with reports on human UPEC (Bengtsson et al.,

267 2012), we found $80 \%$ of the E. coli ST131 isolates to ESBL producers, and all of the ST73

268 isolates to be non-ESBL producers. Overall, our data specified that $15.6 \%$ of UPEC from cats

269 and dogs belong to STs commonly associated with UTI in humans and harbour urovirulence

270 genes that are identical to those found in human isolates (Gibreel et al., 2012). Other STs

271 found in this study, such as ST648 and ST410 are also associated with extraintestinal disease

272 in humans and animals, but not specifically with UTI (Ewers et al., 2014; Falgenhauer et al.

273 2016; Schaufler et al. 2016; Timofte et al., 2016). Therefore, although major UPEC clones

274 described in human infection were not predominant, such strains exhibit reciprocal zoonotic

275 potential, and should be considered a threat to pet owners and companion animals alike.

276 The high prevalence of ST410 in ESBL-producing UPEC from dogs has been observed

277 previously in Switzerland (Huber et al., 2013). This ST, assigned to phylogroup C, displayed

278 inverse association of VF scores with MDR and with plasmid-mediated resistance to third-

279 generation beta-lactams, aminoglycosides and macrolides. This phenomenon was observed

280 among other isolates belonging to phylogenetic groups A and C, suggesting that selective

281 pressure, rather than virulence may be the driving force behind the high prevalence of ST410

282 among canine UPEC. However, the panel of VFs selected for this study was limited in

283 number and represents only a subset of known VFs. Other determinants of virulence may 
have been missed that may have shown a different tendency. Nevertheless, the high

285 prevalence of isolates belonging to phylogenetic groups A, B1 and C indicate that the

286 commensal flora of companion animals may play an important role in the aetiology of UTI.

287 The results of this study illustrate the need for further observation of E. coli causing disease in

288 companion animals, as well as the prudent use of antimicrobials in veterinary medicine,

289 including the use of third generation cephalosporins, fluoroquinolones and macrolides.

\section{Conclusion}

In conclusion, our findings showed that feline and canine UPEC population was characterized

294 by a high prevalence of MDR. ESBLs producing UPEC were detected almost exclusively

295 among canine isolates. Phylogenetic groups B2 and F were associated more frequently with

296 VF than other groups. In this study, group C was the most predominant phylogenetic group

297 and displayed the lowest number of VF. MLST revealed a diverse clonal structure, and

298 detected STs that may pose an infectious threat to humans. The most frequent MLST was

299 ST410, which was characterized by a high prevalence of plasmid-mediated resistance genes

$300 \quad b l a_{\mathrm{CTX}-\mathrm{M}-15}, a a c\left(6^{\prime}\right)-\mathrm{Ib}-\mathrm{cr}$ and $m p h(A)$, and a low aggregate VF score.

301 These results increase our current knowledge of the phenotypic and genotypic characteristics

302 of feline and canine UPEC, and may be advantageous for future infection management and 303 prevention.

\section{Conflict of Interest Statement}

306 Conflicts of interest: none.

\section{$308 \quad$ Funding}


This work was partly supported by the Swiss Federal Office of Public Health, Division

310 Communicable Diseases.

\section{References}

Barsanti, J. 2012. Genitourinary infections. In: Greene C.E. (Ed.), Infectious diseases of the dog and cat. 4th ed. Elsevier Saunders, St Louis, USA, pp. 1013-1031.

Bengtsson, S., Naseer, U., Sundsfjord, A., Kahlmeter, G., Sundqvist, M. 2012. Sequence types and plasmid carriage of uropathogenic Escherichia coli devoid of phenotypically detectable resistance. J. Antimicrob. Chemother. 67, 1, 69-73. DOI=10.1093/jac/dkr421.

Clermont, O., Christenson, J. K., Denamur, E., Gordon, D. M. 2013. The Clermont Escherichia coli phylo-typing method revisited: improvement of specificity and detection of new phylo-groups. Environ. Microbiol. Rep. 5, 1, 58-65. DOI=10.1111/1758-2229.12019.

Clinical Laboratory Standards Institute (CLSI), 2016. Performance Standards for Antimicrobial Susceptibility Testing. 26th ed. CLSI supplement. Clinical Laboratory Standards Institute, Wayne, PA, CLSI document M100S.

Ewers, C., Bethe, A., Stamm, I., Grobbel, M., Kopp, P. A., Guerra, B., Stubbe, M., Doi, Y., Zong, Z., Kola, A., Schaufler, K., Semmler, T., Fruth, A., Wieler, L. H., Guenther, S. 2014. CTX-M-15-D-ST648 Escherichia coli from companion animals and horses: another pandemic clone combining multiresistance and extraintestinal virulence? J. Antimicrob. Chemother. 69, 5, 1224-1230. DOI=10.1093/jac/dkt516.

Falgenhauer, L., Imirzalioglu, C., Ghosh, H., Gwozdzinski, K., Schmiedel, J., Gentil, K., et al. 2016. Circulation of clonal populations of fluoroquinolone-resistant CTX-M-15producing Escherichia coli ST410 in humans and animals in Germany. Int. J. Antimicrob. Agents. 47, 457-465. DOI=10.1016/j.ijantimicag.2016.03.019.

Foxman, B. 2010. The epidemiology of urinary tract infection. Nat. Rev. Urol. 7, 653-660. 
Glaser, C.A., Powers, E.L., Greene, C.E. 2012. Zoonotic infections of medical importance in immunocompromised humans. In: Greene C.E. (Ed.), Infectious diseases of the dog and cat. 4th ed. Elsevier Saunders, St Louis, USA, pp. 1141-1162.

Gibreel, T. M., Dodgson, A. R., Cheesbrough, J., Fox, A. J., Bolton, F. J., Upton, M. 2012. Population structure, virulence potential and antibiotic susceptibility of uropathogenic Escherichia coli from Northwest England. J. Antimicrob. Chemother. 67, 2, 346-356. $\mathrm{DOI}=10.1093 / \mathrm{jac} / \mathrm{dkr} 451$.

Huber, H., Zweifel, C., Wittenbrink, M. M., Stephan, R. 2013. ESBL-producing uropathogenic Escherichia coli isolated from dogs and cats in Switzerland. Vet. Microbiol. 162, 2-4, 992-996. DOI=10.1016/j.vetmic.2012.10.029.

Iranpour, D., Hassanpour, M., Ansari, H., Tajbakhsh, S., Khamisipour, G., Najafi, A. 2015. Phylogenetic groups of Escherichia coli strains from patients with urinary tract infection in Iran based on the new Clermont phylotyping method. Biomed. Res. Int. 2015, 846219. doi:10.1155/2015/846219.

Johnson, J.R. Stell, A. L. 2000. Extended virulence genotypes of Escherichia coli strains from patients with urosepsis in relation to phylogeny and host compromise. J. Infect. Dis. 181, 261-272. DOI=10.1086/315217.

Johnson, J.R., Johnston, B., Clabots, C. R., Kuskowski, M. A., Roberts, E., DebRoy, C. 2008a. Virulence genotypes and phylogenetic background of Escherichia coli serogroup O6 isolates from humans, dogs, and cats. J. Clin. Microbiol. 46, 417-422. DOI=10.1128/JCM.00674-07.

Johnson, J.R., Clabots, C., Kuskowski, M. A. 2008b. Multiple-host sharing, long-term persistence, and virulence of Escherichia coli clones from human and animal household members. J. Clin. Microbiol. 46, 12, 4078-4082. DOI=10.1128/JCM.00980-08.

Johnson, J.R., Owens, K., Gajewski, A., Clabots, C. 2008c. Escherichia coli colonization patterns among human household members and pets, with attention to acute urinary 
tract infection. J. Infect. Dis. 197, 218-224.

Johnson, J.R., Miller, S., Johnston, B., Clabots, C., Debroy, C. 2009. Sharing of Escherichia coli sequence type ST131 and other multidrug-resistant and urovirulent E. coli strains among dogs and cats within a household. J. Clin. Microbiol. 47, 11, 3721-3725. DOI=10.1128/JCM.01581-09.

Kaper, J. B., Nataro, J. P., Mobley, H. L. 2004. Pathogenic Escherichia coli. Nat. Rev Microbiol. 2, 2, 123-140. DOI=10.1038/nrmicro818.

Kaur, J., Chopra, S., Sheevani, G. M. 2013. Modified double disc synergy test to detect ESBL production in urinary isolates of Escherichia coli and Klebsiella pneumoniae. J. Cin. Diagn Res. 7, 229.

Lau, S. H., Reddy, S., Cheesbrough, J., Bolton, F. J., Willshaw, G., Cheasty, T., Fox, A. J., Upton, M. 2008. Major uropathogenic Escherichia coli strain isolated in the northwest of England identified by multilocus sequence typing. J. Clin. Microbiol. 46, 1076-1080.

Lavigne, J.-P., Bruyère, F., Bernard, L., Combescure, C., Ronco, E., Lanotte, P., Coloby, P., Thibault, M., Cariou, G., Desplaces, N. 2016. Resistance and virulence potential of uropathogenic Escherichia coli strains isolated from patients hospitalized in urology departments: a French prospective multicentre study. J. Med. Microbiol. 65, 6, 530-537.

Liu, X., Thungrat,K., Boothe, D.M. 2015. Multilocus sequence typing and virulence profiles in uropathogenic Escherichia coli isolated from cats in the United States. PLoS ONE 10:e0143335. doi: 10.1371/journal.pone.0143335.

Nicolle, L. E. 2013. Antimicrobial resistance in community-acquired Escherichia coli isolated from urinary infection: Good news or bad. Can. J. Infect. Dis. Med. Microbiol. 24, 123124.

Nolan, L. K., Li, G., Logue, C. M. 2015. Origin and dissemination of antimicrobial resistance among uropathogenic Escherichia coli. Microbiol. Spectr. 3, 10.1128/microbiolspec.UTI-0007-2012. 
Nüesch-Inderbinen, M. T., Baschera, M., Zurfluh, K., Hächler, H., Nüesch, H., Stephan, R. 2017. Clonal diversity, virulence potential and antimicrobial resistance of Escherichia coli causing community acquired urinary tract infection in Switzerland. Front. Microbiol. 8, 2334. DOI=10.3389/fmicb.2017.02334.

Ojo, K.K., Ulep, C., Van Kirk, N., Luis, H., Bernardo, M., Leitao, J., Roberts, M. C. 2004. The $m e f(A)$ gene predominates among seven macrolide resistance genes identified in gram-negative strains representing 13 genera, isolated from healthy Portuguese children. Antimicrob. Agents Chemother. 48, 3451-3456. DOI=10.1128/AAC.48.9.34513456.2004.

Olin, S. J. Bartges, J. W. 2015. Urinary tract infections: treatment/comparative therapeutics. Vet. Clin. North Am. Small Anim. Pract. 45, 721-746 $\mathrm{DOI}=10.1016 / \mathrm{j} . \mathrm{cvsm} .2015 .02 .005$.

Schaufler, K., Semmler, T., Wieler, L. H., Wöhrmann, M., Baddam, R., Ahmed, N., Müller, K., Kola, A., Fruth, A., Ewers, C., Guenther, S. 2016. Clonal spread and interspecies transmission of clinically relevant ESBL-producing Escherichia coli of ST410-another successful pandemic clone. FEMS Microbiol. Ecol. 92, 1, 10.1093/femsec/fiv155.

Schmidt, V. M., Pinchbeck, G. L., Nuttall, T., McEwan, N., Dawson, S., Williams, N. J. 2015. Antimicrobial resistance risk factors and characterisation of faecal E. coli isolated from healthy Labrador retrievers in the United Kingdom. Prev. Vet. Med. 119, 31-40. DOI=10.1016/j.prevetmed.2015.01.013.

Spurbeck, R. R., Dinh, P. C., Walk, S. T., Stapleton, A. E., Hooton, T. M., Nolan, L. K., Kim, K. S., Johnson, J. R., Mobley, H. L. T. 2012. Escherichia coli isolates that carry vat, $f y u A, c h u A$, and $y f c V$ efficiently colonize the urinary tract. Infect. Immun. 80, 41154122. DOI=10.1128/IAI.00752-12.

Stegemann, M. R., Passmore, C. A., Sherington, J., Lindeman, C. J., Papp, G., Weigel, D. J., Skogerboe, T. L. 2006. Antimicrobial activity and spectrum of cefovecin, a new 
extended- spectrum cephalosporin, against pathogens collected from dogs and cats in Europe and North America. Antimicrob. Agents Chemother. 50, 2286-2292. DOI=10.1128/AAC.00077-06.

Timofte, D., Maciuca, I. E., Williams, N. J., Wattret, A., Schmidt, V. 2016. Veterinary hospital dissemination of CTX-M-15 extended-spectrum beta-lactamase-producing Escherichia coli ST410 in the United Kingdom. Microb. Drug Resist. 22, 7, 609-615. DOI $=10.1089 / \mathrm{mdr} .2016 .0036$.

Wagner, S., Gally, D. L., Argyle, S. A. 2014. Multidrug-resistant Escherichia coli from canine urinary tract infections tend to have commensal phylotypes, lower prevalence of virulence determinants and ampC-replicons. Vet. Microbiol. 169, 171-178. DOI=10.1016/j.vetmic.2014.01.003.

Wirth, T., Falush, D., Lan, R., Colles, F., Mensa, P., Wieler, L. H., Karch, H., Reeves, P. R., Maiden, M. C. J., Ochman, H., Achtman, M. 2006. Sex and virulence in Escherichia coli: an evolutionary perspective. Mol. Microbiol. 60, 5, 1136-1151. DOI $=10.1111 / j .1365-2958.2006 .05172 . x$.

Woodford, N., Fagan, E. J., ad Ellington, M. J. 2006. Multiplex PCR for rapid detection of genes encoding CTX-M extended-spectrum $\beta$-lactamases. J.Antimicrob.Chemother. 57, 154-155.

Zurfluh, K., Abgottspon, H., Hächler, H., Nüesch-Inderbinen, M., Stephan, R. 2014. Quinolone resistance mechanisms among extended-spectrum beta-lactamase (ESBL) producing Escherichia coli isolated from rivers and lakes in Switzerland. PLoS One. 9, 4, e95864. DOI=10.1371/journal.pone.0095864.

Zurfluh, K., Nüesch-Inderbinen, M., Morach, M., Berner, A. Z., Hächler, H., Stephan, R. 2015. Extended-spectrum ß-lactamase-producing-Enterobacteriaceae in vegetables imported from the Dominican Republic, India, Thailand and Vietnam. Appl. Environ. 
439

440 


\section{$441 \quad$ Figure legend}

442

443 MLST-based eBURST diagram depicting non-ESBL and ESBL producing UPEC isolated

444 from cats and dogs. Each ST is represented by a circle, the size of which correlates to the

445 frequency of the ST. Pie parts represent the number of strains within one ST. Exact numbers

446 of isolates per ST are given in Table 3 and supplementary Table S1. Black outer rings show

447 percentage of dog-derived strains from dogs, white outer rings show percentage of strains

448 from cats. Isolates that tested negative by PCR for bla $a_{\mathrm{ESBL}}$ genes are coloured grey. Isolates

449 harbouring bla $_{\mathrm{CTX}-\mathrm{M}-1}$ are depicted in blue,bla $a_{\mathrm{CTX}-\mathrm{M}-14}$ in red, bla $a_{\mathrm{CTX}-\mathrm{M}-15}$ in orange, bla $_{\mathrm{CTX}-\mathrm{M}-27}$

450 in turquoise, $b_{l a}$ CTX-M-55 in green. 